\title{
A Generalization of the Fuglede-Putnam Theorem to Unbounded Operators
}

\author{
Fotios C. Paliogiannis \\ Department of Mathematics, St. Francis College, 180 Remsen Street, Brooklyn Heights, NY 11201, USA \\ Correspondence should be addressed to Fotios C. Paliogiannis; fpaliogiannis@sfc.edu
}

Received 8 September 2014; Accepted 8 March 2015

Academic Editor: El Hassan Youssfi

Copyright (C) 2015 Fotios C. Paliogiannis. This is an open access article distributed under the Creative Commons Attribution License, which permits unrestricted use, distribution, and reproduction in any medium, provided the original work is properly cited.

Let $N, M$ be unbounded normal operators in a Hilbert space and let $T$ be a closed operator whose domain $\mathscr{D}(T)$ contains the domain of $N$, and the domain $\mathscr{D}\left(T^{*}\right)$ contains the domain of $M$. It is shown that if $T N \subseteq M T$, then $T N^{*} \subseteq M^{*} T$.

\section{Introduction}

In this note we prove a generalization of the classical FugledePutnam theorem to unbounded operators. A special case of this generalization is given in [1]. We begin with some preliminary results.

Let $\mathscr{H}$ be a complex Hilbert space and let $B(\mathscr{H})$ be the algebra of bounded linear operators in $\mathscr{H}$. Let $O p(\mathscr{H})$ denote the set of unbounded densely defined linear operators in $\mathscr{H}$. For $A \in O p(\mathscr{H})$ we denote the domain of $A$ by $\mathscr{D}(A)$. Given $A, B \in O p(\mathscr{H})$, the operator $B$ is called an extension of $A$, denoted by $A \subseteq B$, if $\mathscr{D}(A) \subseteq \mathscr{D}(B)$ and $A x=B x$ for all $x \in$ $\mathscr{D}(A)$. An operator $A \in O p(\mathscr{H})$ is called closed if $A=\bar{A}$ (the closure of $A)$. A closed densely defined operator $A \in O p(\mathscr{H})$ is said to commute with the bounded operator $T \in B(\mathscr{H})$, if $T A \subseteq A T$. This means that for each $x \in \mathscr{D}(A)$, we have $T x \in$ $\mathscr{D}(A)$ and $T A x=A T x$. Let $\{A\}^{\prime}=\{T \in B(\mathscr{H}): T A \subseteq A T\}$. If $A \in B(\mathscr{H})$ this notion agrees with the usual notion of commutant. One sees $\{A\}^{\prime}$ is a strogly closed subalgebra of $B(\mathscr{H})$, and $T \in\{A\}^{\prime}$ if and only if $T^{*} \in\left\{A^{*}\right\}^{\prime}$. Hence, $\{A\}^{\prime} \cap\left\{A^{*}\right\}^{\prime}$ is a von Neumann algebra.

Definition 1. Let $A \in O p(\mathscr{H})$ be closed and $\mathscr{A}$ a von Neumann algebra. If $\mathscr{A}^{\prime} \subseteq\{A\}^{\prime}$, the operator $A$ is said to be affiliated with $\mathscr{A}$, denoted by $A \eta \mathscr{A}$.

The algebra $W^{*}(A)=\left\{\{A\}^{\prime} \cap\left\{A^{*}\right\}^{\prime}\right\}^{\prime}$ is the smallest von Neumann algebra with which $A$ is affiliated, and is referred to it as the von Neumann algebra generated by $A$.
Definition 2. Let $A \in O p(\mathscr{H})$. A bounding sequence for $A$ is a non-decreasing sequence $\left\{F_{n}\right\}_{n \in \mathbb{N}}$ of projections on $\mathscr{H}$ such that $\bigvee_{n=1}^{\infty} F_{n}=I, F_{n} A \subseteq A F_{n}$ and $A F_{n} \in B(\mathscr{H})$ for all $n \in \mathbb{N}$.

Lemma 3 (see [1]). If $\mathscr{A}$ is an abelian von Neumann algebra and $A \eta \mathscr{A}$, then there is a bounding sequence $\left\{F_{n}\right\}$ for $A$ such that $F_{n} \in \mathscr{A}$ and $A F_{n} \in \mathscr{A}$ for all $n \in \mathbb{N}$.

A closed operator $N \in O p(\mathscr{H})$ is normal if $N^{*} N=N N^{*}$. This implies that $\mathscr{D}(N)=\mathscr{D}\left(N^{*}\right)$ and $\|N x\|=\left\|N^{*} x\right\|$ for every $x \in \mathscr{D}(N)$ [2, page 51]. It turns out that the von Neumann algebra $W^{*}(N)$ is abelian, and $W^{*}(N)=\{N\}^{\prime \prime}[3]$. Hence, from Lemma 3 , there is a bounding sequence $\left\{F_{n}\right\}$ for $N$ in $W^{*}(N)$. In fact, $F_{n}=E_{n}-E_{-n}$ for each $n \in \mathbb{N}$, where $\left\{E_{\lambda}\right\}_{\lambda \in \mathbb{R}}$ is the spectral family of the selfadjoint operator $N^{*} N$ [1].

\section{Results}

The Fuglede-Putnam theorem [4] in its classical form states the following.

Theorem 4 (Fuglede-Putnam). Let $N$ and $M$ be normal operators in a Hilbert space. If $T$ is any bounded operator satisfying $T N \subseteq M T$, then $T N^{*} \subseteq M^{*} T$.

The following result from [2, page 97] is essential to our proof of the generalization of the Fuglede-Putnam theorem. 
Lemma 5. Let $A_{1}, A_{2} \in O p(\mathscr{H})$ be self-adjoint operators and let $T \in B(\mathscr{H})$. Then $T A_{1} \subseteq A_{2} T$ if and only if $T E_{\lambda}=P_{\lambda} T$ for all $\lambda \in \mathbb{R}$, where $\left\{E_{\lambda}\right\}_{\lambda \in \mathbb{R}}$ and $\left\{P_{\lambda}\right\}_{\lambda \in \mathbb{R}}$ are the spectral families of $A_{1}$ and $A_{2}$, respectively.

Theorem 6. Let $N, M \in O p(\mathscr{H})$ be normal operators and let $T \in O p(\mathscr{H})$ be a closed operator such that $\mathscr{D}(N) \subseteq \mathscr{D}(T)$ and $\mathscr{D}(M) \subseteq \mathscr{D}\left(T^{*}\right)$. If $T N \subseteq M T$, then $T N^{*} \subseteq M^{*} T$.

Proof. Let $\left\{E_{\lambda}\right\}_{\lambda \in \mathbb{R}}$ and $\left\{P_{\lambda}\right\}_{\lambda \in \mathbb{R}}$ be the spectral families of the self-adjoint operators $N^{*} N$ and $M^{*} M$, respectively. For $m, n \in \mathbb{N}$, consider the bounding sequences $F_{n}=E_{n}-E_{-n}$ and $G_{m}=P_{m}-P_{-m}$ for $N$ and $M$, respectively. Since $\mathscr{D}(N) \subseteq$ $\mathscr{D}(T)$, it follows $\mathscr{H}=\mathscr{D}\left(N F_{n}\right) \subseteq \mathscr{D}\left(T F_{n}\right)$. Since $T F_{n}$ is closed, the closed graph theorem implies $T F_{n} \in B(\mathscr{H})$. Similarly, by the hypothesis on the domain of $M$ and the closed graph theorem, we see $T^{*} G_{m} \in B(\mathscr{H})$.

From the hypothesis $T N \subseteq M T$, we have $T N F_{n} \subseteq M T F_{n}$. Moreover, since $F_{n} N \subseteq N F_{n}$, we also have $T F_{n} N \subseteq T N F_{n}$. Hence,

$$
\left(T F_{n}\right) N \subseteq M\left(T F_{n}\right), \quad \forall n \in \mathbb{N}
$$

Since $T F_{n}$ is bounded, the Fuglede-Putnam theorem implies

$$
\left(T F_{n}\right) N^{*} \subseteq M^{*}\left(T F_{n}\right), \quad \forall n \in \mathbb{N} .
$$

From (1), (2), we have $\left(T F_{n}\right) N^{*} N \subseteq M^{*}\left(T F_{n}\right) N \subseteq$ $M^{*} M\left(T F_{n}\right)$. That is,

$$
\left(T F_{n}\right) N^{*} N \subseteq M^{*} M\left(T F_{n}\right), \quad \forall n \in \mathbb{N} .
$$

Consequently, from Lemma 5,

$$
\left(T F_{n}\right) E_{\lambda}=P_{\lambda}\left(T F_{n}\right), \quad \forall \lambda \in \mathbb{R} .
$$

Therefore

$$
\left(T F_{n}\right) F_{k}=G_{m}\left(T F_{n}\right), \quad \forall k, n, m \in \mathbb{N}
$$

Taking adjoints in (5) we have

$$
\left[G_{m}\left(T F_{n}\right)\right]^{*}=\left[\left(T F_{n}\right) F_{k}\right]^{*}=F_{k}\left(T F_{n}\right)^{*} \supseteq F_{k} F_{n} T^{*} .
$$

But

$$
\left[G_{m}\left(T F_{n}\right)\right]^{*}=\left(T F_{n}\right)^{*} G_{m} \supseteq F_{n} T^{*} G_{m}
$$

As $F_{n} T^{*} G_{m} \in B(\mathscr{H})$, we get

$$
F_{k} F_{n} T^{*} \subseteq F_{n} T^{*} G_{m}
$$

Furthermore, since $F_{n}$ and $F_{k}$ commute,

$$
F_{n} F_{k} T^{*} \subseteq F_{n} T^{*} G_{m}
$$

that is, for every $x \in \mathscr{D}\left(T^{*}\right)$, we have $G_{m} x \in \mathscr{D}\left(T^{*}\right)$ and

$$
F_{n} F_{k} T^{*} x=F_{n} T^{*} G_{m} x
$$

Let $x \in \mathscr{D}\left(T^{*}\right)$ and fix $k, m<n$. Then since $F_{n} \rightarrow I$ (strongly) as $n \rightarrow \infty$, it follows

$$
F_{k} T^{*} \subseteq T^{*} G_{m}, \quad \forall k, m \in \mathbb{N}
$$

Taking adjoints in (11) and using the closeness of $T$,

$$
\left(F_{k} T^{*}\right)^{*} \supseteq\left(T^{*} G_{m}\right)^{*} \supseteq G_{m} T^{* *}=G_{m} \bar{T}=G_{m} T .
$$

But $\left(F_{k} T^{*}\right)^{*}=T^{* *} F_{k}=\bar{T} F_{k}=T F_{n}$. Hence,

$$
G_{m} T \subseteq T F_{k}, \quad \forall k, m \in \mathbb{N} \text {. }
$$

Multiplying (2) by $F_{n}$, we get $\left(T F_{n}\right) N^{*} F_{n} \subseteq M^{*}\left(T F_{n}\right) F_{n}=$ $M^{*} T F_{n}$. Since $\left(T F_{n}\right)\left(N^{*} F_{n}\right)=T N^{*} F_{n}$ and $\left(T F_{n}\right)\left(N^{*} F_{n}\right) \in$ $B(\mathscr{H})$, we obtain

$$
T N^{*} F_{n}=M^{*} T F_{n} \quad \forall n \in \mathbb{N} .
$$

Now let $x \in \mathscr{D}\left(T N^{*}\right)$; that is, $x \in \mathscr{D}\left(N^{*}\right)$ and $N^{*} x \in$ $\mathscr{D}(T)$. Fix $m>k$, and let $m \rightarrow \infty$. Then using (13) and the fact $G_{m} \rightarrow I$ (strongly), we have

$$
T F_{k} x=G_{m} T x \longrightarrow T x .
$$

Moreover, from (14), the fact $F_{n} N^{*} \subseteq N^{*} F_{n}$, and (13), we have

$$
M^{*} T F_{k} x=T N^{*} F_{k} x=T F_{k} N^{*} x=G_{m} T N^{*} x \longrightarrow T N^{*} x .
$$

Since $M^{*}$ is closed, it follows $x \in \mathscr{D}\left(M^{*} T\right)$ and $M^{*} T x=$ $T N^{*} x$. Therefore, $T N^{*} \subseteq M^{*} T$.

As a special case for $M=N$, we obtain the following generalization of Fuglede's theorem [5].

Corollary 7. Let $N \in O p(\mathscr{H})$ be normal and let $T \in O p(\mathscr{H})$ be a closed operator such that $\mathscr{D}(N) \subseteq \mathscr{D}(T) \cap \mathscr{D}\left(T^{*}\right)$. If $T N \subseteq$ $N T$, then $T N^{*} \subseteq N^{*} T$.

Corollary 8. Let $N_{1}, N_{2} \in O p(\mathscr{H})$ be normal operators. If $\mathscr{D}\left(N_{1}\right) \subseteq \mathscr{D}\left(N_{2}\right)$, then $N_{2} N_{1} \subseteq N_{1} N_{2} \Leftrightarrow N_{2} N_{1}^{*} \subseteq N_{1}^{*} N_{2}$.

Corollary 9. Let $N, N_{1}, N_{2} \in O p(\mathscr{H})$ be normal operators. If $\mathscr{D}\left(N_{i}\right) \subseteq \mathscr{D}(N)$, for $i=1,2$, then $N N_{1} \subseteq N_{2} N \Leftrightarrow N N_{1}^{*} \subseteq$ $N_{2}^{*} N$.

Remark 10. Recently in the article "An All-UnboundedOperator Version of the Fuglede-Putnam Theorem," Complex Analysis and Operator Theory (2012) [6: 1269-1273], a similar result was offered, but its proof is incorrect. In fact, on the last page of this paper [page 1273] the proof is wrong; note that from the equality $P_{B_{m}}(M) A N^{*} P_{B_{n}}(N) x=$ $P_{B_{m}}(M) M^{*} A P_{B_{n}}(N) x$, the fact $P_{B_{m}}(M) \rightarrow I$ (strongly) gives $A N^{*} P_{B_{n}}(N) x=M^{*} A P_{B_{n}}(N) x$; however, (dealing with unbounded operators, as is the case here) the fact (alone) that $P_{B_{n}}(N) \rightarrow I$ (strongly) does not give the equality $A N^{*} x=$ $M^{*} A x$.

\section{Conflict of Interests}

The author declares that there is no conflict of interests regarding the publication of this paper. 


\section{References}

[1] F. C. Paliogiannis, "A note on the Fuglede-Putnam theorem," Proceedings-Mathematical Sciences, vol. 123, no. 2, pp. 253256, 2013.

[2] K. Schmündgen, Unbounded self-adjoint operators in Hilbert space, vol. 265 of Graduate Texts in Mathematics, Springer, 2012.

[3] F. C. Paliogiannis, "On Fuglede's theorem for unbounded normal operators," Ricerche di Matematica, vol. 51, no. 2, pp. 261-264 (2003), 2002.

[4] C. R. Putnam, "On normal operators in Hilbert space," American Journal of Mathematics, vol. 73, pp. 357-362, 1951.

[5] B. Fuglede, "A commutativity theorem for normal operators," Proceedings of the National Academy of Sciences of the United States of America, vol. 36, pp. 35-40, 1950. 


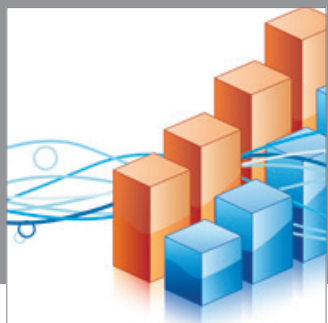

Advances in

Operations Research

mansans

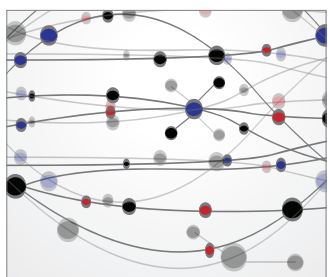

The Scientific World Journal
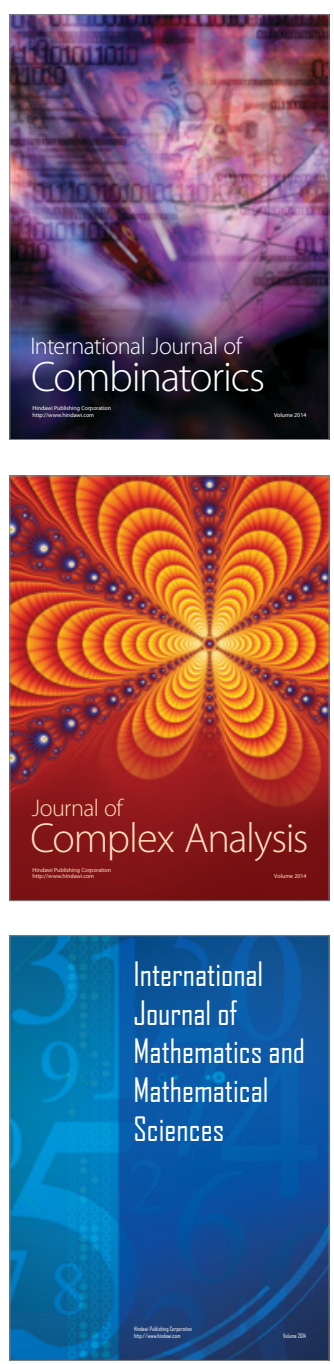
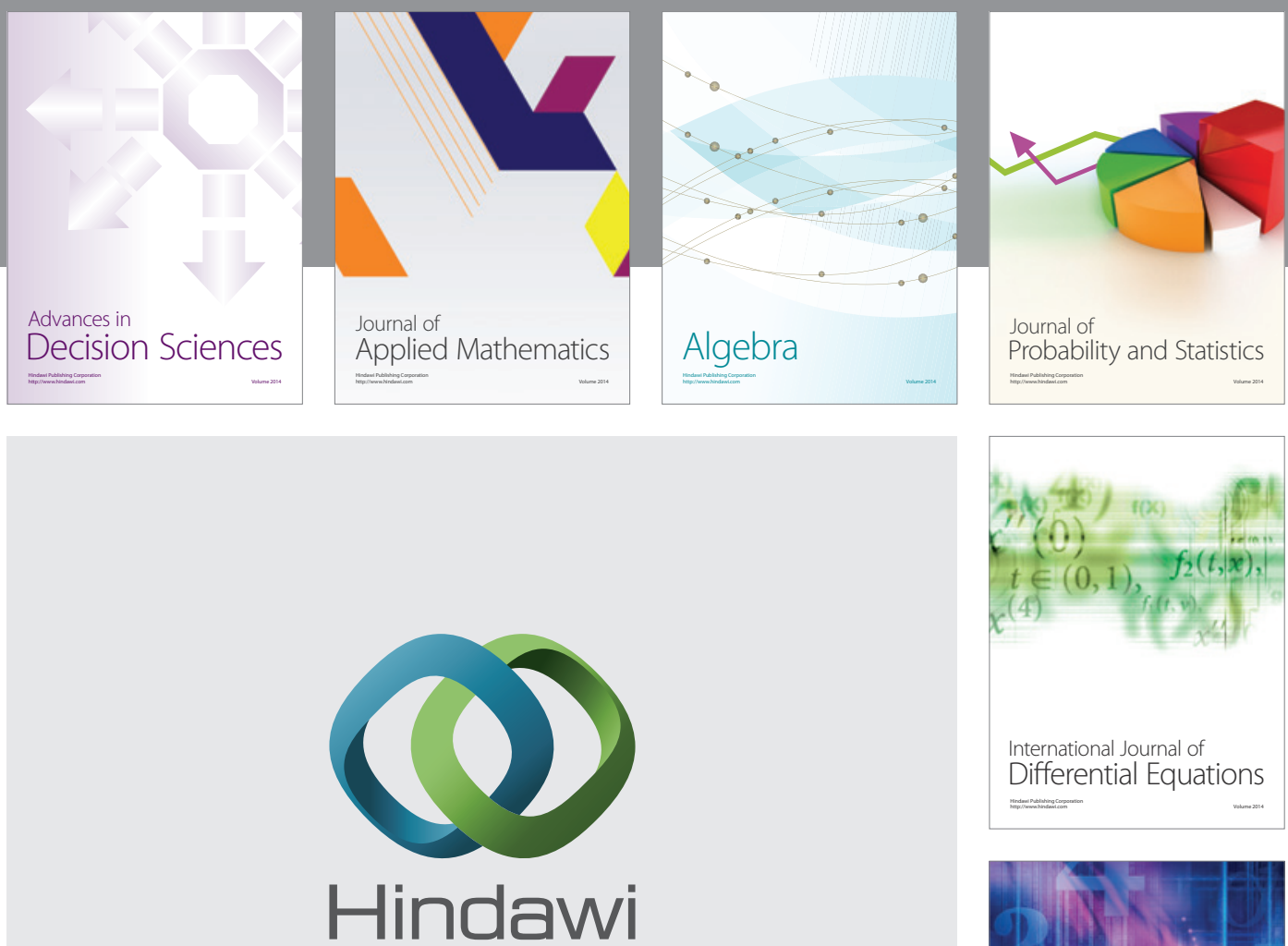

Submit your manuscripts at http://www.hindawi.com
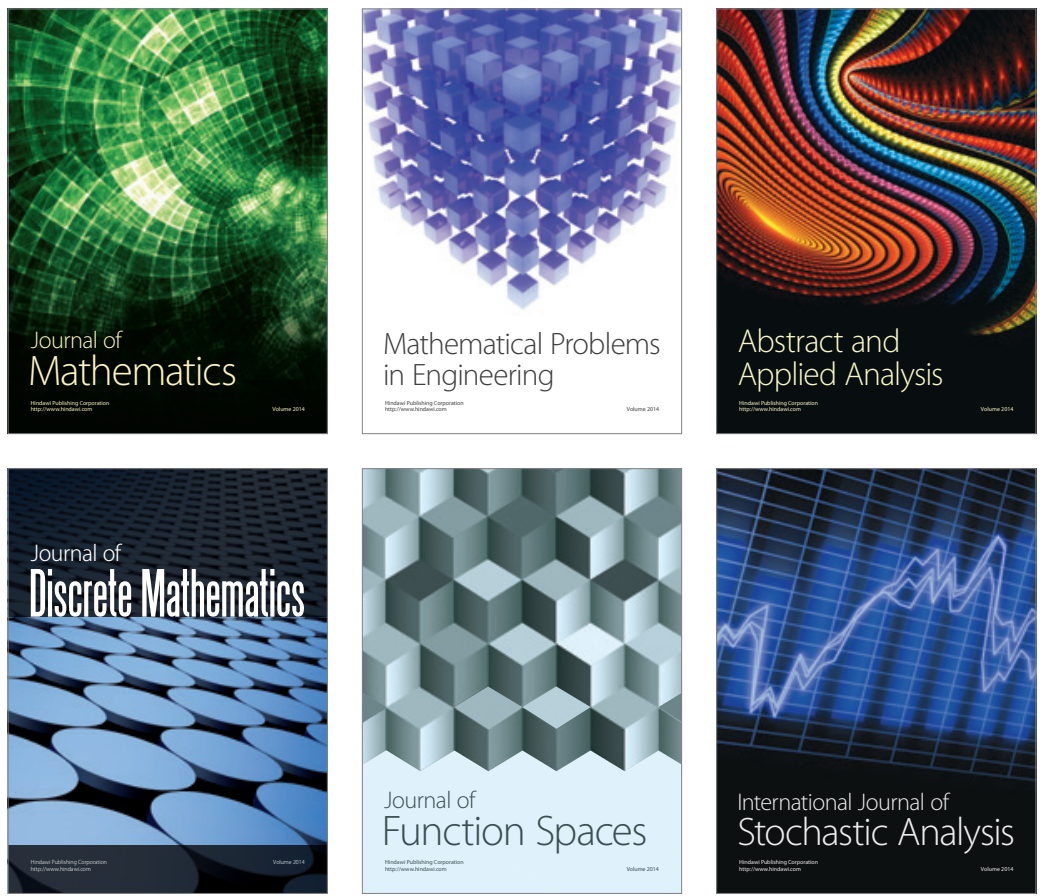

Journal of

Function Spaces

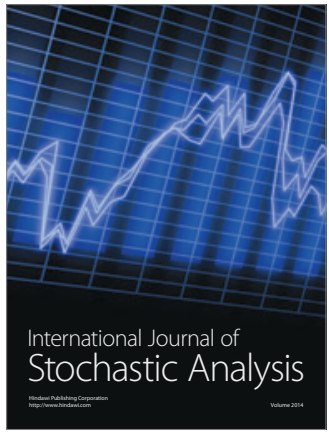

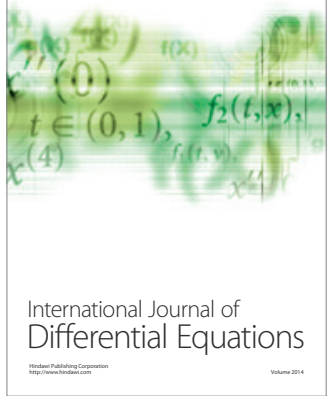
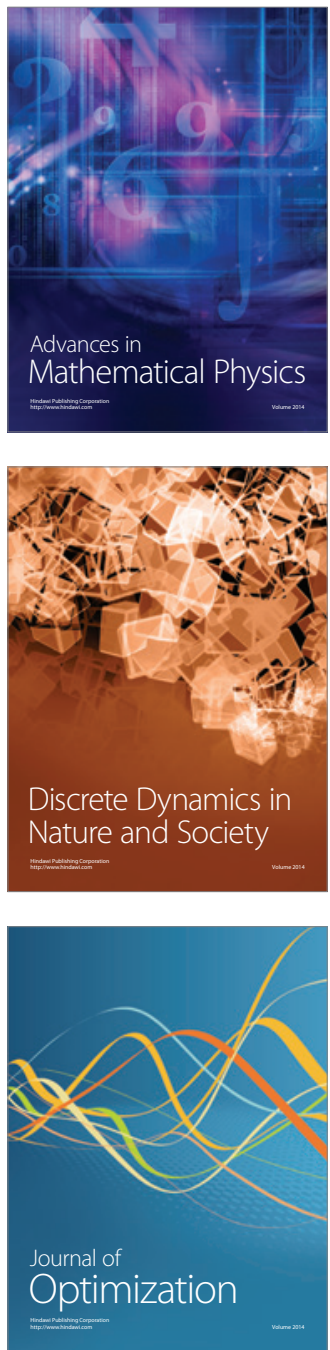